\title{
VIVÊNCIAS DE RESIDENTES ENFERMEIROS NO PROGRAMA DE RESIDÊNCIA MULTIPROFISSIONAL EM SAÚDE
}

\author{
EXPERIENCES OF RESIDENT NURSES ON THE RESIDENCY \\ PROGRAM IN MULTIPROFESSIONAL HEALTH
}

\author{
Rhea Silvia de Avila Soares', Marcia Aparecida Penna', Camila Pinno', Vânia Lúcia Durgante', \\ Alexsandra Michelini Real Saul', Elaine Miguel Delvivo Farão², Suzinara Beatriz Soares de Lima ${ }^{3}$
}

\begin{abstract}
RESUMO
Compreender as vivências o residente enfermeiro acerca de suas práticas em Programa de Residência Multiprofissional Integrada em Sistema Público de Saúde, desenvolvido em Hospital Universitário do Estado do Rio Grande do Sul. Estudo com abordagem qualitativa e coleta de dados por meio de entrevista semiestruturada, realizada no período de dezembro de 2012 à fevereiro de 2013, sendo os sujeitos da pesquisa enfermeiros residentes do $1^{\circ} \mathrm{e} 2^{\circ}$ ano do programa de residência. Participaram desta pesquisa 07 residentes enfermeiros. Para análise dos dados utilizou-se a análise de conteúdo de Bardin, pela qual se identificou a categoria: Atuação do residente enfermeiro: percepções acerca de sua prática e formação multiprofissional, dividida em duas subcategorias: Identificação com as propostas do programa de residência multiprofissional e Vivências/Sentimentos despertados a partir das atividades práticas. Os resultados indicam que na visão dos residentes a formação pela residência multiprofissional potencializa o pensar-agir para uma assistência integral a saúde no âmbito do SUS e ainda revelam dificuldades na compreensão a respeito da integração ensino-serviço na efetivação do SUS.
\end{abstract}

Descritores: Enfermagem; Formação Continuada; Internato e Residência.
${ }^{1}$ Graduada em Enfermagem pela Universidade Federal de Santa Maria (UFSM), Santa Maria, RS, Brasil.

${ }^{2}$ Mestre em Enfermagem pela Universidade Federal de Minas Gerais (UFMG), Belo Horizonte, MG, Brasil.

${ }^{3}$ Doutora pela Universidade Federal de Santa Maria (UFSM), Santa Maria, RS, Brasil.

\begin{abstract}
Identify the understanding of resident nurses about their practices in Residence Program Integrated Multi Professional in Public Health System, developed at the University Hospital of the State of Rio Grande do Sul. Study with qualitative approach and data collection by means of semi-structured interviews, conducted during the period December 2012 to February, condubeing the subject of the research nurses residents of the 1 st and 2 nd year of the residency program. Participated in this research 07 resident nurses. For data analysis we used the content analysis of Bardin, by which identified the category: Performance of resident nurses: perceptions about their practice and formation multiprofessional, divided into two subcategories: Identification with the proposals of the residency program multiprofessional and Experiences/Feelings awakened from practical activities. The results indicate that in the point of view of the resident training by multiprofessional residency potentiates the think-act for an integral assistance to health under the SUS and also reveal difficulties in understanding about teaching-service integration in the execution of the SUS.
\end{abstract}

Descriptors: Nursing; Nurses; Education Continuing; Internship and Residency. 


\section{Introdução}

No ano de 1976, a Secretaria do Estado de Saúde do Rio Grande do Sul criou a primeira Residência em Medicina Comunitária no Brasil, denominada São José do Murialdo, que mais tarde passaria a ser considerada multiprofissional' ${ }^{1}$. As Residências Multiprofissionais em Saúde (RMS) são cursos de pós-graduação, lato sensu, regulamentadas pela promulgação da lei n. 11.129, de 30 de junho de $2005^{2}$, destinada aos profissionais que integram a área de saúde, excluída a medicina ${ }^{3}$.

Com a promulgação da Constituição Federal no ano de 1988, inicia-se um processo de mudança com relação a criação de um sistema de saúde mais justo e igualitário. Assim, instaura-se a formação do Sistema Único de Saúde (SUS), como um sistema púbico, universal e descentralizado. ${ }^{4,5}$ Percebe-se a necessidade de mudanças no modelo de atenção à saúde como uma exigência social, que interelaciona, tanto a população usuária do SUS, quanto os profissionais de saúde e pesquisadores ${ }^{1}$.

As RMS tem como objetivo formar residentes para uma atuação sustentada por meio de uma concepção ampliada de saúde e ancorada pelos Princípios e Diretrizes do SUS ${ }^{6,7}$. Ainda, buscam formar profissionais com habilidades e especialidades específicas conforme a deficiência regional ${ }^{7}$, estimulando competências para o desenvolvimento de ações de gestão e atenção, viabilizando novos cenários integrados e descentralizados de ensino-aprendizagem. Também, destaca-se o caráter potencializador na integração de ações de ensino-serviço, proporcionando um espaço de troca de saberes e experiência, a partir de uma assitência interdisciplinar ${ }^{6}$.

Uma importante característica da RMS está relacionada a interdisciplinaridade, a qual estimula uma formação coletiva "inter-categorias" com valorização dos "núcleos" de saberes específicos de cada profissão, contribuindo para a integralidade do cuidado1. Ressalta-se que existe uma dificuldade nos projetos políticos pedagógicos (PPP) com relação aos conceitos de multiprofissional, interprofissional, multidisciplinar e interdisciplinar, o que comprova-se em estudo que analisou os PPP dos Programas de Residência Multiprofissional em Saúde (PRMS) do Estado de São Paulo, e revelou que os programas apresentam diversidade de termos relacionados aos prefixos multi e inter e aos adjetivos profissional e disciplinar ${ }^{8}$.

O PPP que orienta e conduz o processo formativo dos residentes que fazem parte do cenário deste estudo, apresenta os conceitos de interdisciplinaridade, intersetorialidade e interinstitucionalidade ${ }^{6}$. Destaca que existe uma interação profissional ineficiente, fragmentada e mecanicista entre as equipes de trabalho que compõe os diferentes níveis de complexidade do sistema de saúde. Assim, a proposta deste programa de residência é a integração de ações disciplinares, intersetoriais e interinstitucionais, a fim de alcançar a integralidade nos vários níveis do sistema na perspectiva do SUS6.

O Ministério da Saúde, desde 2003, tem estimulado os cursos de RMS, contrapondo-se ao ensino de práticas exclusivamente curativas, enfatizando o trabalho interdisciplinar, contribuindo na construção de novos saberes e fazeres que promovam uma atenção mais humana e integral à saúde da população $0^{3,6,7}$.

A Portaria $n^{0} 1.111$ de 05 de julho de 2005, designa ao preceptor a função de supervisão docente-assistencial, realizada por área de atuação ou especialidade profissional. São responsáveis pela orientação dos residentes nas atividades de núcleo e de campo estimulando a integração dos diferentes saberes por meio da interdisciplinaridade.,7,8 Corroborando, 0 Decreto $n^{0}$ 94.406, de 08 de junho de 1986 que regulamenta a Lei $n^{0} 7498$, do exercício profissional do enfermeiro, afirma que os enfermeiros podem participar dos programas de treinamento e aprimoramento de pessoal de saúde 9 .

A motivação para a realização deste estudo originou-se durante as atividades de preceptoria de núcleo na área de enfermagem em um Programa de Residência Multiprofissional Integrada em Sistema Público de Saúde (PRMISPS) vinculada a uma Universidade Federal da Região Sul do país. As discussões dos preceptores com os residentes nas reuniões de preceptoria de núcleo, são momentos que servem para discutir a prática, aprofundar conhecimentos e ainda sanar as dúvidas do dia a dia. Desta maneira, estas discussões suscitaram questionamentos sobre a atuação do residente enfermeiro no programa de residência multiprofissional.

Diante do exposto e buscando nortear este estudo propõe-se as seguintes questões: As ações propostas pelo Programa de Residência Multiprofissional para o profissional enfermeiro estão sendo alcançadas? Como o residente enfermeiro compreende sua prática no programa de residência multiprofissional? Portanto, o objetivo do presente estudo é compreender as vivências do residente enfermeiro em um Programa de Residência Multiprofissional Integrada em Sistema Público de Saúde. 


\section{Metodologia}

Este estudo caracteriza-se como uma pesquisa exploratória com abordagem qualitativa, desenvolvido em um Hospital Universitário localizado no Estado do Rio Grande do Sul, referência de média e alta complexidade para região centro-oeste do estado. Este hospital é local de atuação dos residentes do Programa de Residência Multiprofissional Integrada em Sistema Público de Saúde, com ênfase na atenção hospitalar.

A oferta de vagas para o programa acontece por área de concentração e por núcleo profissional. As áreas de concentração na atenção hospitalar dividem-se em: saúde mental, hemato-oncologia, materno-infantil e crônicodegenerativo, com vagas para enfermagem, nutrição, serviço social, terapia ocupacional, fisioterapia, fonoaudiologia e farmácia e odontologia ${ }^{10}$.

Atualmente, o núcleo de enfermagem possui duas vagas para área de onco-hematologia, uma vaga para maternoinfantil, duas para área crônico-degenerativo e três para saúde-mental, totalizando a entrada de oito enfermeiros por ano no hospital ${ }^{10}$.

A coleta de dados ocorreu por meio de entrevista semiestruturada de dezembro de 2012 à fevereiro de 2013, sendo os sujeitos da pesquisa enfermeiros residentes do $1^{\circ}$ e $2^{\circ}$ ano do programa de residência do ano de 2012.

Assim, foram incluídos na pesquisa os enfermeiros residentes que estavam cursando $01^{\circ}$ ou $2^{\circ}$ ano da residência multiprofissional e excluídos os que estavam de licença de qualquer natureza.

A população do estudo no período de coleta de dados era de 15 enfermeiros residentes. Aplicados os critérios de inclusão e critérios de exclusão, a pesquisa constituiu-se de sete participantes, uma vez que: duas enfermeiras estavam em licença maternidade, duas enfermeiras de férias e quatro residentes enfermeiras participaram como autoras do projeto.

Os participantes foram convidados por contato telefônico para participarem da pesquisa, sendo agendado encontro individual para a coleta de dados que aconteceu em sala da residência localizada no hospital. As entrevistas foram gravadas por meio de aparelho digital de gravação e posteriormente transcritas.

Dessa forma, foram coletadas sete entrevistas, que foram iniciadas por meio da seguinte questão: "Fale de sua vivência e sua prática, enquanto residente enfermeiro, no Programa de Residência Multiprofissional Integrada". Foi realizada análise descritiva da primeira parte do instrumento de coleta de dados composto por questões sociodemográficas caracterizando os sujeitos.

Para avaliação dos dados, utilizou-se a análise de conteúdo de Bardin ${ }^{11}$. A partir deste método, os dados foram analisados e interpretados. Para a análise das entrevistas transcritas na íntegra, utilizou-se uma ficha de extração de dados para cada entrevista, nesta ficha registraram-se as principais unidades de análise ou significado, através do registro de sentenças, frases ou parágrafos das transcrições.

Após fichamento de extração de dados, iniciou-se a codificação cromática dos achados fichados, com a utilização de cores para agrupar as unidades de significado de acordo com seu grau de proximidade, a partir do objetivo proposto pela pesquisa. Terminada a codificação cromática, foram elaboradas categorias e subcategorias temáticas através deste ir e vir ao material, das fichas de extração de dados codificadas e criatividade dos pesquisadores.

A realização desta pesquisa obteve aprovação do Comitê de Ética em Pesquisa com Seres Humanos, registrado com o $n^{0}$ da CAEE 07381512.8.0000.5346, garantindo aos participantes confidencialidade e sigilo dos dados conforme os termos da Resolução 196/96 do Conselho Nacional de Saúde, resolução vigente na época em que se desenvolveu a pesquisa $^{12}$. Assim, as falas dos residentes foram identificadas pela letra $E$ seguida de número ordinal na ordem em que as entrevistas foram acontecendo.

\section{Resultados e Discussão}

Participaram desta pesquisa 07 residentes enfermeiros que possuíam entre 23 e 24 anos, todas do sexo feminino, sem filhos, duas com experiência profissional, quatro com experiência no SUS durante a graduação, três com especialização lato sensu, quatro com conhecimento do Projeto Político Pedagógico (PPP) da residência.

A análise dos dados identificou a categoria: Atuação do residente enfermeiro: percepções acerca de sua prática e formação multiprofissional, dividida em três subcategorias: Identificação com as propostas do programa de residência multiprofissional, Conhecimento do Projeto Político Pedagógico (PPP) pelos enfermeiros residentes e Vivências/ sentimentos despertados a partir das atividades práticas. 
A residência multiprofissional é um diferencial na formação do residente enfermeiro uma vez que a atuação com diferentes núcleos profissionais permite um olhar mais ampliado sobre o usuário e o processo de trabalho dos diferentes núcleos, produzindo mudanças no sentido de atender a população de forma integral.

\section{Atuação do residente enfermeiro: Percepções acerca de sua prática e formação multiprofissional}

O enfermeiro residente deve promover o gerenciamento do cuidado de forma interdisciplinar, buscando a integralidade da assistência em sua área de concentração e contribuindo para uma articulação com os demais serviços que compõe a rede de atenção à saúde, conforme o projeto que orienta o programa de residência ${ }^{6}$.

Nessa perspectiva, considerando que o SUS tem como um dos seus princípios a integralidade a Residência Multiprofissional potencializa o desenvolvimento desse princípio pois contribui para a assistência em uma linha de cuidado integral, de forma interdisciplinar, direcionando o fluxo do usuário no sistema de saúde ${ }^{13-14}$.

Esta perspectiva se reproduz na fala dos residentes enfermeiros participantes desta pesquisa:

É um programa que tem toda a questão de unir a prática, a teoria, a experiência profissional, ter uma ampla visão de como funciona o SUS, todo este processo de rede, troca de experiência com outros profissionais (E2).

Dessa forma, a formação no programa de residência contribui para a sedimentação de conhecimentos adquiridos na graduação, possibilitando o crescimento do profissional na articulação entre os conceitos teóricos aprendidos acerca do SUS e sua empregabilidade no cotidiano da prática.

Assim, os profissionais formados por residências multiprofissionais em saúde, possuem condições críticas ampliadas, possibilitando uma atuação diferenciada na busca pela integralidade da assistência, seja na formação de outros profissionais em atividades de docência, como na prática profissional tanto na rede pública como suplementar . $^{6}$

A atuação profissional proposta pela residência pressupõe a articulação entre serviços e ações de saúde com outras políticas públicas, como forma de assegurar a construção de caminhos para a integralidade da assistência, com repercussão mais efetiva na saúde e qualidade de vida dos indivíduos, conforme evidenciado na fala a seguir:

A carga horária complementar na Secretária Municipal de Saúde é muito positivo [...] a questão da gestão, de tu estar com os técnicos responsáveis pelas políticas públicas, verifica-se que tudo é planejado conforme as necessidades (E2).

Sob essa perspectiva, compreende-se a importância da atuação dos residentes enfermeiros nas atividades complementares realizadas nas unidades gestoras como a Secretaria Municipal de Saúde, o que oportuniza a integração das instituições de ensino e serviços de saúde, na perspectiva do fortalecimento do SUS e das Políticas públicas de saúde vigentes no País.

Além disso, as práticas de gestão possibilitam a disseminação de conhecimentos acerca da necessidade de implementação de projetos que ampliem os espaços na busca de uma assistência interdisciplinar, articulada em rede visando a integralidade da assistência.

Nesta perspectiva, os profissionais formados por programas que possibilitem práticas de gestão, poderão ser líderes atuantes na mudança dos paradigmas práticos que conformam a atuação no sistema público de saúde ${ }^{6}$.

\section{Identificação com as propostas do programa de residência multiprofissional}

O PRMISPS tem como cenário de formação a gestão e a atenção hospitalar, sendo buscada, pelo programa, a formação de profissionais com competências teóricas, práticas e crítico-reflexivas para atuar no Sistema Público de Saúde ${ }^{6,13}$.

Norteado pelos Princípios e Diretrizes do SUS ${ }^{12}$, este programa busca a instituição de mecanismos que possibilitem a integração das ações e dos serviços no sistema de saúde; tendo como objetivo desenvolver nos profissionais residentes capacidades para atuarem de modo não apenas interdisciplinar, mas também intersetorial, efetivando práticas que viabilizem um novo modo de pensar-fazer que integre o cenário de intervenção Hospitalar com os diferentes níveis de complexidade do sistema público de saúde ${ }^{6,14}$. 
Corroborando, os residentes relatam em suas falas ações interdisciplinares, intersetoriais e interinstitucionais:

Trazer para a equipe, para unidade, para o serviço, a proposta de que os profissionais trabalham em conjunto, que discutimos os casos juntos, criando um vinculo maior uma relação com a atenção básica (E7).

Uma atuação diferenciada, mais multiprofissional, mais junto com o paciente, mais discussões com os colegas para ver o que resolver em cada caso (E3).

As RMS caracterizam-se pela interdisciplinaridade, com diversas categorias profissionais da área da saúde. Vi sa à formação coletiva em equipe, tendo como objetivo principal mudança do modelo de saúde, que ainda é predominantemente centrado na doença e em alguns profissionais da saúde; e principalmente visa à transformação do processo de trabalho em saúde, por meio de uma visão crítico-reflexiva ${ }^{15}$.

Tendo em vista a superação de modelos limitados ao binômio queixa-conduta, diagnóstico e tratamento, os profissionais da saúde precisam ser formados com capacidade para inovar seu processo de trabalho, pensando em novas formas de atuação e que contemplem, também, as necessidades e as demandas dos usuários ${ }^{16,17}$.

Neste sentido, a assistência multiprofissional é uma das estratégias implementadas no SUS para garantir a integralidade no cuidado, a partir da relação entre diferentes profissões que atuam de forma articulada e integrada com intuito de alcançar uma maior efetividade das ações em saúde ${ }^{16}$.

O que pode ser retratado na fala do participante entrevistado:

Na residência tu tem atividades de núcleo, relativas a tua atuação profissional como enfermeiro, e as atividades de campo, que é a relação multiprofissional, mas que tu vai continuar desempenhando funções do enfermeiro (E5).

Assim, verifica-se que nas atividades dos residentes são desenvolvidos atributos que acompanharão o profissional no desempenho de sua prática de núcleo, tendo em vista a busca pela integralidade da assistência ${ }^{6}$. Dessa forma, é necessário que se multipliquem, no campo da saúde, a construção de práticas que considerem múltiplos saberes interconectados, a fim de dar conta da complexidade do cuidado e visando a efetivação dos princípios SUS.

\footnotetext{
A residência foca nas linhas de cuidado, a parte do núcleo e de tu trabalhar também em campo (E6).

[...] relacionar a residência com o todo de forma multidisciplinar, trabalhando com os outros núcleos de profissões integrando no serviço (E7).
}

A inserção do residente enfermeiro no PRMS busca potencializar o trabalho com outros núcleos profissionais a partir das atividades interdisciplinares desenvolvidas, buscando efetivar na prática cotidiana a ampliação do olhar profissional para além do enfoque biologicista.

Para tanto, faz se necessário que os profissionais inseridos no programa, sejam capazes de reconhecer e compreender suas funções. Dessa forma, os residentes enfermeiros foram questionados sobre o PPP da residência, 0 que originou a subcategoria que segue:

\section{Conhecimento do Projeto Político Pedagógico (PPP) pelos enfermeiros residentes}

Acerca do conhecimento relacionado ao PPP, os enfermeiros residentes demonstraram pouco ou nenhum conhecimento, como evidenciado nas falas:

Pouca coisa, só o que nos passam nas aulas, mas eu nunca li, na residência a gente acaba fazendo mais coisas do serviço (E3).

Eu cheguei a ler o manual, antes de entrar na residência, mas não tenho um conhecimento muito profundo, aos poucos quando eu entrei fui me integrando (E7). 
Assim, é demonstrado nos dados, que mesmo conhecendo pouco o PPP da residência, os enfermeiros buscam sanar esse desconhecimento na medida em que são integrados no programa e identificam suas competências neste processo de formação. Nesta linha de pensamento, percebe-se que compreendem o diferencial de suas ações nas falas a seguir quando identificam a importância de trabalhar com projeto terapêutico singular, clínica ampliada, interdisciplinaridade, integralidade da assistência a partir da referência e contra referência, ambos propostos como práticas do enfermeiroresidente no PPP do Programa6.

Dispositivos assim como o Projeto Terapêutico Singular, ente outros né, clínica ampliada [...] a gente consegue atuar em grupo e fazer algum projeto, em um ambulatório (E1).

No momento que a gente assume o plantão a gente é responsável pela unidade e a gente desenvolve todas as competências legais do enfermeiro [...] e as atividades multiprofissionais [...] costumo dizer que o enfermeiro é multiprofissional por natureza (E5).

A vivência do residente enfermeiro inserido em uma RMISPS marca e determina o processo de formação e possui significado importante na construção da identidade profissional do residente, o que fica evidenciado na subcategoria encontrada a seguir.

\section{Vivências/sentimentos despertados no residente enfermeiro a partir das atividades práticas}

Vários sentimentos, sensações, expectativas se misturam durante este período de formação. Os residentes enfermeiros identificam suas competências como exposto na subcategoria acima, mas nem sempre encontram facilidades neste processo, enfrentam também algumas dificuldades. Estas facilidades e dificuldades despertam sentimentos impactantes na formação e na qualidade de vida deste sujeito, como amadurecimento profissional, felicidade, medo, angústia, incerteza.

As falas a seguir relatam as facilidades identificadas e os sentimentos vivenciados:

Onde tem preceptor parece que o serviço flui melhor, onde tem uma pessoa que tem visão da residência, do que é o projeto, enfim do que seriam nossas funções aqui (E1).

É muito satisfatório, apesar dos conflitos e dificuldades de adaptação, é possivel ser muito feliz na residência (E5).

A oportunidade de crescimento, tudo é crescimento, cada dia a gente aprende mais [...] estou feliz com este momento (E7).

Os residentes apontaram como um facilitador no processo de formação a atuação dos preceptores, que são profissionais responsáveis pelo acompanhamento do desempenho do residente em suas atividades teórico práticas. Dessa forma, possuem importância singular na inserção do residente nos campos de prática, sendo os mediadores das relações, dos conflitos e embates existentes ${ }^{6,12}$.

As atividades de preceptoria são divididas em preceptorias de campo e de núcleo, sendo ambas significativas nos dados apontados, para a implementação do projeto proposto pelo programa de residência ${ }^{6}$, como retratado no dado que segue:

Visão do SUS, questão teórica que é bem importante [...] as aulas foram bem boas [...] bem importante ter este embasamento teórico, todas as reflexões. As preceptorias que a gente tem de núcleo e de campo [...] acho bem valido (E2).

A necessidade de mediar conflitos, provém da proposta da residência de instaurar novos processos de trabalho que contribuam para uma clínica ampliada, sob um olhar interdisciplinar. Porém, não se trata de tarefa simples essa execução, sendo explanados, a seguir, dados que denotam dificuldades encontradas, bem como sentimentos que emergiram destas:

A gente faz muito núcleo, muita assistência, assistência e assistência, não se consegue ter uma visão do todo em alguns momentos (E1). 
No inicio tive dificuldades na parte técnica, procedimentos de enfermagem, no entanto o maior problema foi nas relações humanas (E4).

Minhas expectativas são um pouco frustradas em relação de não conseguir um trabalho mais ampliado, trabalho de equipe [...] da unidade não entender qual é o nosso papel na residência (E2).

A partir dos relatos supracitados constata-se que as equipes podem não estar preparadas para receber estes profissionais de acordo com as propostas da residência, sendo este um desafio para o desenvolvimento das atividades do residente.

Ao encontro destes achados, autores descrevem a inserção de residente enfermeiro em unidade de clínica ${ }^{19}$, que relata o desconhecimento do papel das residentes de enfermagem em uma unidade de internação e a dificuldade de sensibilização em relação à residência multiprofissional.

Assim, faz se necessário a realização de planejamentos cada vez mais articulados entre os programas de residência multiprofissionais e os serviços que recebem estes residentes, bem como a valorização dos preceptores como fundamentais para que transformações duradouras aconteçam nos serviços.

\section{Considerações Finais}

Com a realização desta pesquisa, entende-se que há um desafio a ser conquistado em relação à formação e consequente, vivência dos enfermeiros residentes, com vistas à interdisciplinaridade, a mudança na lógica de cuidados e a inserção desses sujeitos nos diversos cenários de atenção à saúde. Busca-se pelo Programa de Residência Multiprofissional integrar teoria e prática em uma perspectiva interdisciplinar de formação, cujo objetivo principal é a mudança do modelo de saúde, que ainda é predominantemente centrado na doença.

Percebe-se que o residente enfermeiro busca potencializar sua formação com outros núcleos profissionais, a partir das atividades baseadas na prática profissional. Essas atividades possibilitam uma reflexão crítica, a busca de informação e do conhecimento, com possibilidade de transformar a prática e promover a saúde dos indivíduos. No entanto, verifica-se que, inicialmente, ao ingressarem no Programa alguns desconhecem o PPP, porém, ao se inserirem nos campos de prática identificam a importância de trabalhar com a lógica da integralidade na assistência, a necessidade de permanente articulação entre os setores de atenção e a importância da negociação entre 0 individual e 0 coletivo, características próprias da interdisciplinaridade.

Como facilitadores para o processo de formação, referenciam a importância dos preceptores nas atividades teórico práticas, que promovem a ambientação e facilitam suas vivencias nos locais de prática com a equipe de trabalho locoregional e, entre os diferentes serviços de atenção. A atuação sensível do preceptor parece diminuir entre os residentes e a equipe dos serviços os sentimentos de frustração, incerteza e desamparo.

Dessa forma, o desafio de melhoria e construção diária do SUS está posto, mas novas ferramentas também estão sendo construídas, dentre elas, a construção de Programas de Residência Multiprofissionais comprometidos com a formação do sujeito para uma atenção integral, efetiva, compromissada com o sujeito para além da prática conservadora, mas numa perspectiva de humanização efetiva de busca diária de qualidade nos serviços no âmbito do SUS.

\section{Referências}

1. Paiva, CHA, Teixeira, LA. Reforma sanitária e a criação do Sistema Único de Saúde: notas sobre contextos e autores. História, Ciências, Saúde - Manguinhos, Rio de Janeiro, 2014 Jan/Mar; 21 (1): 15-35

2. Nascimento DG do, Oliveira $M A$ de $C$. Competências profissionais e o processo de formação na residência multiprofissional em saúde da família. Saúde Soc. 2010; 19(4): 814-827.

3. Rosa SDR, Lopes RE. Residência multiprofissional em saúde e pós-graduação lato sensu no Brasil: apontamentos históricos. Trab. Educ. Saúde. 2010; 7(3): 479-98.

4. Ministério da Saúde (BR), Secretaria de Gestão do Trabalho e da Educação na Saúde, Departamento de Gestão da Educação na Saúde. Residência multiprofissional em saúde: experiências, avanços e desafios. Brasília: Ministério da Saúde, 2006. 
5. Ministério da Saúde (Brasil). Portaria n 1.111, de 5 de julho de 2005. Fixa normas para a implementação e a execução do Programa de Bolsas para a Educação pelo Trabalho. Diário oficial da união 05 de julho 2005.

6. Universidade Federal de Santa Maria. Centro de Ciências da Saúde, Hospital Universitário de Santa Maria, $4^{a}$ Coordenadoria Regional de Saúde-RS, Secretaria de Município da Saúde de Santa Maria. Residência multiprofissional integrada em gestão e atenção hospitalar no sistema público de saúde. Santa Maria; 2009. Disponível em: Disponível em: http://jararaca.ufsm.br/websites/residenciamulti/Resid.mult/ProjMS1.pdf> Acesso em 09 de abril de 2014.

7. Cheade, MFM et al. Residência multiprofissional em saúde: a busca pela integralidade. Cogitare Enferm. 2013 Jul/Set; 18(3):592-5

8. Miranda Neto MV, Leonello VM, Oliveira MAC. Multiprofessional residency in health: a document analysis of political pedagogical projects. Rev Bras Enferm. 2015;68(4):586-93. DOI: http://dx.doi.org/10.1590/0034-7167.2015680403i

9. BRASIL. Lei n 7.498/86, de junho de 1986. Dispõe sobre a regulamentação do exercício da Enfermagem e dá outras providências. Diário Oficial [da] República Federativa do Brasil, Brasília, DF; 1986.

10. Universidade Federal de Santa Maria (UFSM), http://coral.ufsm.br/residenciamulti/, 2016. Acesso em 16 de março de 2016.

11. Bardin L. Análise de conteúdo. São Paulo: Edições 70; 2011.

12. Ministério da Saúde, Conselho Nacional de Saúde, Comissão Nacional de Ética em pesquisa. Resolução no 196, de outubro de 1996. Aprovar diretrizes e normas regulamentadoras de pesquisas envolvendo seres humanos. Diário Oficial da União 10 out 1996; Seção 1

13. Brasil. Lei n 8080, de 19 de setembro de 1990. Lei orgânica da saúde. Dispõe sobre as condições para a promoção, proteção e recuperação da saúde, a organização e o funcionamento dos serviços correspondentes e dá outras providências. Diário Oficial [da] República Federativa do Brasil, Brasília, DF; 1990.

14. Oliveira MS. Inserção da residência multiprofissional em saúde da família e comunidade no contexto da graduação dos cursos da área de saúde. In: Ministério da Saúde, Secretaria de Gestão do Trabalho e da Educação na Saúde, Departamento Gestão da Educação em Saúde. Residência multiprofissional em saúde: experiências, avanços e desafios. Brasília: Ministério da Saúde; 2006. p. 123-140.

15. Soares RS de A, Penna MA, Farão EMD Engel RH, Soster CC, et al. A atuação do Enfermeiro em um programa de Residência Multiprofsissional em saúde. Biblioteca Lascasas, 2013. 9(1): 103-114.

16. Vial SEM, Plein FB, Machado ME. (Coord.). A política de educação permanente em saúde: avanços e desafios. Porto Alegre: Secretaria de Estado da Saúde do Rio Grande do Sul/Escola de Saúde Pública; 2010.

17. Salvador A de S, Medeiros C da S, Cavalcanti PB, Carvalho RN de. Construindo a multiprofissionalidade: um olhar sobre a residência multiprofissional em saúde da família e comunidade, Rev. bras. ciênc. saúde. 2011; 15 (3):329-338.

18. Guimarães TG. Papel do preceptor na residência multiprofissional: experiência da nutrição. Porto Alegre: Universidade Federal do Rio Grande do Sul. Faculdade de Educação. Curso de Especialização em Práticas Pedagógicas para a Educação em Serviços de Saúde; 2010.

19. Drago LC, Salum RL, Andrade SR, Medeiros M, Marinho MM. A inserção do residente em enfermagem em uma unidade de internação cirúrgica: práticas e desafios. Cogitare Enferm. 2013:18(1): 95-101. 


\section{Rhea Silvia de Avila Soares}

Endereço para correspondência - Rua: Antônio Botega, ap.202 , n 913 ,

Bairro: São José, CEP: 97095-030, Santa Maria, RS, Brasil.

E-mail: rheasilviasoares@yahoo.com.br

Lattes: http://lattes.cnpq.br/7766499332487272

Marcia Aparecida Penna - marciaapenna@ymail.com

Camila Pinno - camilapinno@yahoo.com.br

Vânia Lúcia Durgante - vaniadurgante@yahoo.com.br

Elaine Miguel Delvivo Farão - elainebiofis@yahoo.com.br

Alexsandra Michelini Real Saul - alexsandrarsaul@hotmail.com

Suzinara Beatriz Soares de Lima - suzibslima@yahoo.com.br

Enviado em 05 de Abril de 2016. Aceito em 05 de Julho de 2016. 